\title{
High-resolution magnified endoscopy combined with flexible spectral imagining colour enhancement techniques in the diagnosis of Helicobacter pylori disease
}

\author{
Omer Burcak Binicier ${ }^{1}$, Gozde Dervis Hakim ${ }^{1}$, Sadiye Mehtat Unlu², Omer Selahattin Topalak ${ }^{3}$ \\ ${ }^{1}$ Department of Gastroenterology, Tepecik Education and Research Hospital, Izmir, Turkey \\ 2Department of Pathology, Faculty of Medicine, Dokuz Eylul University, Izmir, Turkey \\ ${ }^{3}$ Department of Gastroenterology, Faculty of Medicine, Dokuz Eylul Universıty, Izmir, Turkey
}

Gastroenterology Rev 2019; 14 (3): 202-210

DOI: https://doi.org/10.5114/pg.2019.88170

Key words: Helicobacter pylori, intestinal metaplasia, endoscopy.

Address for correspondence: Omer Burcak Binicier MD, Department of Gastroenterology, Tepecik Education and Research Hospital, Izmir, Turkey, e-mail: binicieromer@yahoo.com

\begin{abstract}
Aim: To evaluate the efficiency of the flexible spectral imaging colour enhancement (FICE) procedure added to high-resolution magnified endoscopy (HRME) for the evaluation of structural changes in gastric mucosa infected with Helicobacter pylori (H. pylori).

Material and methods: A total of 104 patients were included in the study. First HRME and then HRME + FICE methods were used for the evaluation of the gastric mucosal structure, and examined areas were studied histopathologically. Mucosal appearance was evaluated with the modified Yagi classification. Images were shown to five endoscopists in order to examine inter- and intra-observer variability in image assessment.

Results: Sensitivity and specificity of the image pattern noted with HRME in the antrum in the detection of $H$. pylori were $67.9 \%$ and $84.6 \%$, respectively, while these were $93.5 \%$ and $92.3 \%$, respectively, with HRME + FICE. Type 5 pattern in the antrum, which we thought to be associated with intestinal metaplasia, was not observed in any patient with HRME. Sensitivity and specificity values of type 5 pattern noted with HRME + FICE technique for intestinal metaplasia were $50 \%$ and $98.8 \%$, respectively.

Conclusions: The results show that HRME + FICE as a digital chromoendoscopic method provided an additional diagnostic contribution to HRME for showing the presence of H. pylori and intestinal metaplasia and is a method with higher sensitivity and specificity. The "patchy appearance" (type 5) observed in the antrum not previously described in the evaluations with FICE can be a guiding sign especially for the diagnosis of intestinal metaplasia.
\end{abstract}

\section{Introduction}

Detection of lesions, differential diagnosis by identifying changes in mucosal pattern, as well as the correlation between imaging and histopathology are among the main purposes of diagnostic gastrointestinal system endoscopy. Many systems with proven efficiency have been developed in the last two decades in gastrointestinal system imaging, following the rapidly progressing technological developments. Among these are high-resolution endoscopes, magnified endoscopes, chromoendoscopy, fluorescence imaging, endocytoscopy, confocal laser endoscopy (CLE), and digital filter techniques.
In the studies, the efficiency of these new endoscopic imaging techniques comprises a wide spectrum from gastrointestinal system disorders to diagnosis of Barrett's epithelium, detection of colon polyps, diagnosis of early stomach cancer, and surveillance of ulcerative colitis [1-8]. The place of newly developed endoscopic methods in many other gastrointestinal system lesions is being investigated. Endoscopic imaging techniques like Narrow Band Imaging (NBI), i-Scan, and Multi-Band Imaging $(\mathrm{MBI})$ are being used increasingly, and methods defined as digital chromoendoscopy enhance the imaging of the vascular network and tissue structure of the mucosa and 
allow characterisation, discrimination, and diagnosis of tissues [9]. These techniques transform the surface structure of the mucosa into virtual newly organised imaging structures by using selected wavelengths thanks to digital imaging software systems. Previous studies have shown the correlation between images obtained with magnified chromoendoscopy and NBI methods and normal gastric mucosal structure and gastritis $[4,10,11]$.

\section{Aim}

The purpose of this study is to evaluate the gastric mucosal structure and presence of Helicobacter pylori (H. pylori) with high-resolution magnified endoscopy (HRME) and magnified flexible spectral imaging colour enhancement (FICE) (HRME + FICE) technique.

\section{Material and methods}

\section{Study subjects}

Patients over 18 years old, with complaints of dyspepsia, iron deficiency anaemia, and abdominal pain, who agreed to participate in the study were chosen by Dokuz Eylul University Faculty of Medicine Department of Gastroenterology Endoscopy Unit. Among those, patients were selected according to the presence of lesions detected by upper gastrointestinal endoscopy, not by conventional endoscopy.

Exclusion criteria are listed below:

1. Patients with coagulopathy or bleeding diathesis

2. Pregnant or lactating patients

3. Patients who have used antibiotics within the last month

4. Patients who have used proton pump inhibitors and $\mathrm{H} 2$ receptor antagonists within the last 15 days

5. Patients who have used aspirin, non-steroidal antiinflammatory drugs, or clopidogrel within the last 15 days

6. Patients with severe co-morbidities

7. Patients who have undergone gastric surgery

8. Detection of an ulcer, erosion, polyps, or the presence of cancer in the stomach or small intestines during the procedure

Consent was obtained from the Ethics Committee of Clinical and Laboratory Research of Dokuz Eylul University Faculty of Medicine with approval number 2011/33-05.

\section{Methods}

\section{Upper gastrointestinal system endoscopy}

Patients underwent upper gastrointestinal system endoscopy following a fasting period of at least $8 \mathrm{~h}$, under sedoanalgesia, without any supplemental medication or use of image quality enhancing agents during the procedure with HRME (model EG-490ZW5, Fujinon, Fuji Photo Optical Co., Ltd., Saitama, Japan). In order to obtain a good quality image during the procedure and to prevent migration of gastric contents to the examined area, a transparent head was placed at the tip of the endoscope. Following standard video endoscopic examination of the oesophagus, stomach, and duodenum, unless there was a dominant area regarding appearance, the area of antrum $3 \mathrm{~cm}$ from the pylorus in the direction of the smaller curvature and the area in the distal of the corpus at $3 \mathrm{~cm}$ from the corpus-antrum junction in the direction of the greater curvature were examined in high resolution. Following evaluation of relevant areas with magnified endoscopy and recording of pictures, the same areas were examined by switching the system to FICE with the aid of a button on the endoscope. At least three images were taken for each of the 10 FICE channels recorded in the computer system. Following imaging, one biopsy each was taken from these examined areas in the corpus and antrum for rapid urease test and pathologic diagnosis.

\section{Evaluation and classification with HRME and FICE}

The mucosal pattern of the antrum and corpus was evaluated with the modified Yagi classification shown in Table I. In 2007, Yagi et al. modified their former Z classification and created the A-B classification based on the combination of microsurface and microvascular patterns [12].

\section{Rapid urease test (RUT)}

Biopsy samples taken during endoscopy were placed in 2-ml of RUT solution. The RUT solution is obtained by dissolving $1 \mathrm{~g}$ urea in $10 \mathrm{ml}$ distilled water and two drops of $1 \%$ phenol red are dropped on the $2 \mathrm{ml}$ solution. Following addition of the biopsy sample to the described solution, if the colour of biopsy and/or solution turns red within $5 \mathrm{~min}$, RUT is considered to be positive. RUT from the antrum and corpus were studied separately.

\section{Pathologic examination}

Biopsy samples were placed in $10 \%$ formalin solution and sent to the pathology laboratory, where they underwent routine processing and were paraffinated. Tissue sections were dyed with haematoxylin and eosin, Giemsa, Alcian blue, and Periodic acid-Schiff and were then examined. Classification of gastritis was made according to the revised Sydney System [8].

\section{Statistical analysis}

The Statistical Package for Social Sciences (SPSS) version 15.0 was used for statistical data analysis. Con- 
Table I. Characteristics of mucosal patterns in gastric mucosa (modified Yagi classification)

\begin{tabular}{|c|c|}
\hline Antrum & \\
\hline Type 1 & Mucosa with needle-like pits on a flat surface \\
\hline Type 2 & Spiral sub-epithelial capillary network (SECN) pattern and reticular mucosal microscopic surface of the mucosa \\
\hline Type 3 & Irregular and coarse granular structure of the mucosa \\
\hline Type 4 & Increase in the number of villi and papillary pattern \\
\hline Corpus & \\
\hline B-0 & $\begin{array}{c}\text { Regular sized round pits (in crypt opening: } \mathrm{CO} \text { ), and regularly distributed collective venules (CV) in a star-like structure } \\
\text { covered with regular sub-epithelial capillary (SEC) nets }\end{array}$ \\
\hline B-1 & $\begin{array}{l}\text { Circular pits distributed regularly or in a slightly irregular pattern. Pits are surrounded by the SECN similar to honeycomb } \\
\text { shape, and collector venules are not observed }\end{array}$ \\
\hline B-2 & Round pits and sulcus separating them, no normal SECN and collector venules \\
\hline B-3 & Lesser pits and denser sulcus, mucosa with abnormal SECN and abnormal collector venules \\
\hline A-1 & Sparse and bent SEC on a swollen surface area \\
\hline A-2 & Villous or granular tissue appearance with irregularly narrowed and twisted SEC \\
\hline
\end{tabular}

tinuous variables were expressed as mean and standard deviation, and categorical variables as percentages. The Kruskal Wallis test was used for inter-group comparisons and the Mann-Whitney $U$ test for dual-group comparisons. $\chi^{2}$ test in table format was used in the comparison of categorical variables. After sensitivity, specificity, positive predictive values (PPV), and negative predictive values (NPV) were calculated regarding the methods, data were presented within 95\% confidence intervals. Kappa analysis was carried out to evaluate the diagnostic compatibility of the two methods. A $p$-value $<0.05$ was considered to be significant.

\section{Results}

\section{Characteristics of the patient group}

Causes like ulcer, polyps, and cancer were detected with endoscopy in the stomach and duodenum in

Table II. Demographic characteristics of patients according to the presence of $\mathrm{H}$. pylori in the antrum

\begin{tabular}{|c|c|c|}
\hline Parameter & $\begin{array}{l}\text { H. pylori (+) } \\
(n=78)\end{array}$ & $\begin{array}{l}\text { H. pylori (-) } \\
(n=26)\end{array}$ \\
\hline Age $($ mean $\pm S D)$ & $46.1 \pm 11.8$ & $45.2 \pm 13.0$ \\
\hline \multicolumn{3}{|l|}{ Gender: } \\
\hline Female & $59(75.6 \%)$ & $20(76.9 \%)$ \\
\hline Male & 19 (24.4\%) & $6(23.2 \%)$ \\
\hline \multicolumn{3}{|l|}{ Endoscopy indication: } \\
\hline Dyspepsia & $33(42.3 \%)$ & $10(38.5 \%)$ \\
\hline Iron deficiency anaemia & $18(23.1 \%)$ & $9(34.6 \%)$ \\
\hline Abdominal pain & $23(29.5 \%)$ & $5(19.2 \%)$ \\
\hline
\end{tabular}

H. pylori-Helicobacter pylori.
23 of 127 patients who met inclusion and exclusion criteria and agreed to participate in our study after giving written consent; therefore, they were not taken for examination.

Of the 104 patients included in the study, 76\% (79) were female and the mean age was $45.9 \pm 12.08$ (21-65 years) years.

Helicobacter pylori gastritis was detected in 78 (75\%) patients in the antrum and in 72 (69.2\%) patients in the corpus. Intestinal metaplasia was present in the antrum in 15 patients (14.4\%) and in the corpus in 16 (15.3\%) patients.

Demographic data of the patients and indications for endoscopy according to the presence of $\mathrm{H}$. pylori in the antrum and corpus are given in Tables II and III. When the demographic data of 104 patients included in the study were analysed regarding the presence of H. pylori in the antrum and corpus, there were no statistically significant differences among the groups in terms of age, gender, and reason for ordering endoscopy (Tables II, III).

Atrophy was seen in only 5 of the patients included in the study; therefore, it was not included in the evaluation because significant statistical data could not be obtained in this group.

\section{Comparison of HRME and HRME + FICE regarding $H$. pylori gastritis of the antrum}

\section{HRME}

Histologically and according to the results considered together with RUT results, $H$. pylori was detected in 25 of 47 patients with type 1 and 2 appearances considered to be normal antrum mucosal patterns with 
HRME. Helicobacter pylori was positive in 53 of $57 \mathrm{pa}$ tients with type 3, 4, and 5 appearances, which are considered to be abnormal mucosal patterns. Statistically significant differences were found between patients with normal and abnormal mucosal patterns evaluated with HRME regarding Hp positivity $(p<0.001)$. Patients with abnormal mucosal appearances had higher rates of $H$. pylori positivity. The sensitivity of the procedure in the detection of $H$. pylori was $67.9 \%$ and specificity was $87.6 \%$. Its positive predictive value (PPV) was $92.9 \%$ (95\% Cl: 77.0-95.0) and negative predictive value (NPV) 46.8\% (95\% Cl: 27.6-57.8) (Table IV).

\section{$H R M E+F I C E$}

Histologically and according to the results considered together with RUT results, $H$. pylori was detected in 5 of 29 patients with type 1 and 2 appearances considered to be normal antrum mucosal patterns with HRME + FICE. Helicobacter pylori was positive in 73 of 75 patients with type 3, 4, and 5 appearances, which are considered to be abnormal mucosal patterns. Statistically significant differences were found between patients assessed as having normal and abnormal mucosal pat- terns evaluated with HRME + FICE imaging regarding H. pylori positivity $(p<0.001)$. Similar to HRME, patients with abnormal mucosal appearances had higher rates of $H$. pylori positivity. The sensitivity of the procedure in the detection of $H$. pylori was $93.5 \%$ and specificity was 92.3\%. PPV was 97.3\% (95\% Cl: 82.9-96.9) and NPV was $82.7 \%$ (95\% Cl: 45.3-80.7) (Table IV).

Table III. Demographic data of patients according to the presence of $H$. pylori in the corpus

\begin{tabular}{lcc} 
Parameter & $\begin{array}{c}\text { H. pylori }(+) \\
(\boldsymbol{n}=\mathbf{7 2})\end{array}$ & $\begin{array}{c}\text { H. pylori }(-) \\
(\boldsymbol{n}=\mathbf{3 2})\end{array}$ \\
\hline Age (mean \pm SD) & $46.4 \pm 11.4$ & $44.6 \pm 13.5$ \\
\hline \begin{tabular}{l} 
Gender: \\
\hline Female
\end{tabular} & $54(75 \%)$ & $25(78.1 \%)$ \\
\hline Male & $18(25 \%)$ & $7(21.9 \%)$ \\
\hline Endoscopy indication: & & \\
\hline Dyspepsia & $31(43.05 \%)$ & $15(46.9 \%)$ \\
\hline Iron deficiency anaemia & $19(26.39 \%)$ & $9(28.1 \%)$ \\
\hline Abdominal pain & $22(30.56 \%)$ & $8(25 \%)$ \\
H. pylori-Helicobacter pylori. & &
\end{tabular}

Table IV. Utility of HRME and HRME + FICE methods regarding the presence of H. pylori, intestinal metaplasia, and chronic inflammation

\begin{tabular}{|c|c|c|c|c|}
\hline Parameter & Sensitivity (\%) & Specificity (\%) & PPV (\%) & NPV (\%) \\
\hline \multicolumn{5}{|l|}{ Antrum } \\
\hline \multicolumn{5}{|l|}{ H. pylori: } \\
\hline HRME & 67.9 & 87.6 & 92.9 & 46.8 \\
\hline HRME + FICE & 93.5 & 92.3 & 97.3 & 82.7 \\
\hline \multicolumn{5}{|c|}{ Intestinal metaplasia: } \\
\hline \multicolumn{5}{|l|}{ HRME } \\
\hline HRME + FICE & 50 & 98.8 & 88.8 & 91.5 \\
\hline \multicolumn{5}{|c|}{ Chronic inflammation: } \\
\hline HRME & 68 & 79.3 & 89 & 48.9 \\
\hline $\mathrm{HRME}+\mathrm{FICE}$ & 94.6 & 86.2 & 94.6 & 86.2 \\
\hline \multicolumn{5}{|l|}{ Corpus: } \\
\hline \multicolumn{5}{|l|}{ H. pylori: } \\
\hline HRME & 88.9 & 62.5 & 84.2 & 71.4 \\
\hline $\mathrm{HRME}+\mathrm{FICE}$ & 98.6 & 75 & 88.8 & 96.0 \\
\hline \multicolumn{5}{|c|}{ Intestinal metaplasia: } \\
\hline HRME & 100 & 89.9 & 33.3 & 100 \\
\hline HRME + FICE & 92.3 & 96.7 & 80 & 98.8 \\
\hline \multicolumn{5}{|c|}{ Chronic inflammation: } \\
\hline HRME & 90 & 75 & 90.7 & 75 \\
\hline HRME + FICE & 100 & 89.2 & 96.2 & 100 \\
\hline
\end{tabular}

H. pylori-Helicobacter pylori, PPV - positive predictive value, NPV - negative predictive value, HRME - high-resolution magnified endoscopy, HRME + FICE high-resolution magnified endoscopy + flexible spectral imaging colour enhancement. 
Comparison of HRME and HRME + FICE regarding $H$. pylori gastritis of the corpus $H R M E$

Histologically and according to the results considered together with RUT results, H. pylori was detected in eight of 28 patients with BO appearances, which are considered to be normal corpus mucosal patterns with HRME. Helicobacter pylori was positive in 64 of 76 patients with $\mathrm{B} 1, \mathrm{~B} 2, \mathrm{~B} 3, \mathrm{~A} 1$, and $\mathrm{A} 2$ appearances, which are considered to be abnormal mucosal patterns. Statistically significant differences were found between patients evaluated as having normal and $a b$ normal mucosal patterns evaluated with HRME imaging regarding $H$. pylori positivity $(p<0.001)$. Similarly to that in the antrum, patients with abnormal mucosal appearances had higher rates of $H$. pylori positivity. Sensitivity of the procedure in the detection of $H$. pylori was $88.9 \%$ and specificity was $62.5 \%$, PPV was 84.2\% (95\% Cl: 66.2-86.2), and NPV was 71.4\% (95\% Cl: 29.4-67.4) (Table IV).

\section{HRME + FICE}

Histologically and according to the results considered together with RUT results, H. pylori was detected in one of 25 patients with B0 appearances considered to be normal corpus mucosal patterns with HRME + FICE. H. pylori was positive in 71 of 79 patients with B1, B2, $B 3, A 1$, and $A 2$ appearances, which are considered to be abnormal mucosal patterns. Statistically significant differences were found between patients evaluated as having normal and abnormal mucosal patterns evaluated with HRME + FICE endoscopic imaging regarding H. pylori positivity $(p<0.001)$. Similar to antrum data, patients with abnormal mucosal appearances had higher rates of $H$. pylori positivity. The sensitivity of the procedure in the detection of $H$. pylori was $98.6 \%$, specificity was $75 \%$, PPV was $88.8 \%(95 \% \mathrm{Cl}: 67.1-86.2)$, and NPV was $96 \%$ (95\% Cl: 34.5-76.7) (Table IV).

\section{Comparison of HRME and HRME + FICE regarding intestinal metaplasia of the antrum}

\section{$H R M E$}

Signs of intestinal metaplasia were noted histologically in 6 of 47 patients with type 1 and 2 appearances considered as normal antrum mucosal patterns, with magnified endoscopy. In 10 of 57 patients with type 3 and 4 appearances considered as abnormal mucosal patterns and signs of intestinal metaplasia were found histologically. Type 5 pattern, considered to be specific for intestinal metaplasia, could not be shown in any patient with magnified endoscopy.

\section{$H R M E+F I C E$}

Signs of intestinal metaplasia were found histologically in one of 29 patients with type 1 and 2 appearances, considered as normal antrum mucosal patterns, with HRME + FICE. In seven of 66 patients with type 3 and 4 appearances, considered as abnormal mucosal patterns, signs of intestinal metaplasia were found histologically. Type 5 pattern, considered to be specific for intestinal metaplasia, was observed in nine patients, and signs of intestinal metaplasia were shown histologically in eight of these patients. The sensitivity of the procedure in detecting intestinal metaplasia in the antrum was $50 \%$, specificity was $98.8 \%$, PPV was $88.8 \%$ (95\% Cl: $39.2-$ 61.9), and NPV was $91.5 \%$ (95\% Cl: 61.2-94.9) (Table IV).

\section{Comparison of HRME and HRME + FICE regarding intestinal metaplasia of the corpus}

HRME

Signs of intestinal metaplasia were not found histologically in 29 of 29 patients with BO appearances, considered as normal corpus mucosal patterns, with HRME. Five of 15 patients with intestinal metaplasia had A1 or A2 patterns, while one had B1, two had B2, and seven had B3 patterns. The sensitivity of the procedure in detecting intestinal metaplasia in the corpus was $100 \%$, specificity was $89.9 \%$, PPV was $33.3 \%$ (95\% Cl: 42.2-97.5), and NPV was 100\% (95\% Cl: 84.396.3) (Table IV).

\section{HRME + FICE}

Signs of intestinal metaplasia were not found histologically in 25 of 25 patients with B0 appearances, considered as normal corpus mucosal patterns, with HRME + FICE. Twelve of 15 patients with intestinal metaplasia had $\mathrm{A} 1$ or $\mathrm{A} 2$ patterns, one had $\mathrm{B} 1$, and two had $\mathrm{B} 3$ patterns. PPV was $80 \%(95 \% \mathrm{Cl}: 41.3-88.8)$ and NPV was $98.8 \%$ (95\% Cl: 90.3-99.2) (Table IV).

As summarised in Table IV, correlation of HRME + FICE technique with histopathological signs and presence of $H$. pylori in both the antrum and corpus was higher than that of HRME. In the evaluation of the gastric mucosa, the diagnostic contribution of magnified endoscopy used together with $\mathrm{MBI}$ for the presence of $H$. pylori was better than the HRME only technique. For the presence of $H$. pylori in the antrum, sensitivity and specificity values of evaluating with HRME were $67.9 \%$ and $87.6 \%$, while these rates were $93.5 \%$ and $92.3 \%$ with the HRME + FICE technique, respectively. Sensitivity and specificity values for detecting $\mathrm{H}$. pylori in the corpus with HRME were $88.9 \%$ and $62.5 \%$, while these rates were $98.6 \%$ and $75 \%$ with HRME + FICE, respectively. 
When the two methods were compared regarding diagnosis, a statistically significant difference was found both for antrum and corpus $(p<0.001)$. More abnormal mucosal patterns were identified with FICE. However, when the compatibility of two methods was evaluated as the presence or absence of an abnormality for the antrum and corpus, it was at an acceptable level ( $\kappa$ for antrum: 0.478, $\kappa$ for corpus: 0.621).

\section{Discussion}

With the widespread use of upper gastrointestinal system endoscopy with advanced technique, signs that are detected with endoscopy, such as erythema, granularity, fragility, oedema, effacement or prominence of gastric rugae, presence of visible vessels, and fine white mucosal deposits, were thought to be associated with diagnoses like gastritis, $H$. pylori, intestinal metaplasia, and atrophy [10, 13-15]. However, there are still relatively few published studies describing the endoscopic features of $\mathrm{H}$. pylori-induced gastritis. In developing countries, the prevalence of $H$. pylori is around $80 \%$ and is currently similar to that in Turkey [16]. In this study, the presence of $H$. pylori in 104 patients without gastric or duodenal ulcers was found to be $78 \%$. In far east countries where gastric cancer is common, rates of atrophy are around $30 \%$ and intestinal metaplasia $25 \%$ [17]. We found the rate of intestinal metaplasia in the antrum and corpus to be around 15\%. Particularly in populations like our country, with high prevalence of $H$. pylori, detection of $H$. pylori proven to have an association with precancerous lesions with cheaper and accessible methods is of great importance. Demonstration of $\mathrm{H}$. pylori presence and intestinal metaplasia with endoscopic appearance patterns gains importance in terms of saving costs and time. Similar to chromoendoscopic HRME studies showing good correlation between gastric histopathological results and the presence of $H$. pylori, in our study carried out to evaluate gastric mucosal structure and $H$. pylori presence with HRME and the addition of FICE to HRME using modified Yagi classification, antiperistaltic or proteolytic agents were not used before the procedure in order to be similar to daily practice. Evaluation of antrum and corpus was carried out using a single classification, which was modified Yagi classification, and not separately for mucosal vascularity and microstructure.

When the compatibility of the two methods was evaluated regarding the presence or absence of an abnormality in the antrum and corpus, it was seen to be at an acceptable level ( $\kappa$ for antrum: $0.478, \kappa$ for corpus: 0.621).

In the Anagnostopoulos et al. study they found the specificity of type 2 and type 3 patterns for detecting an $\mathrm{H}$. pylori infection with HRME to be $92.7 \%$ and $100 \%$, respectively [18]. In the Yagi et al. study, the specificity of type 3 pattern with HRME for the predicting of H. pylori gastritis was found to be $96.2 \%$ [19]. We found similar results to those of Anagnostopoulos et al. and Yagi et al. in our study; $H$. pylori was detected in all of the cases found to have type 4 pattern with HRME in the antrum and in more than $90 \%$ of the cases with type 3 pattern. Similarly, H. pylori was seen in all of the cases found to have type 4 pattern in the antrum with HRME + FICE technique and in nearly all of the cases with type 3 pattern. In other words, there is no need for sampling toward $H$. pylori in the detection of type 3 and 4 patterns in both HRME and HRME + FICE techniques.

Redéen et al. found sensitivity and specificity of moderate atrophic gastritis in the corpus with HRME to be $67 \%$ and $48 \%$, respectively [13]. In our study, in the detection of B1-2 and 3 appearances, considered to be signs of inflammation, similarly in the corpus, the presence of $H$. pylori was seen to be above $90 \%$ both with HRME and HRME + FICE, and the presence of these appearances should suggest the presence of $H$. pylori with both techniques. These results are similar to the Anagnostopoulos et al. study which found that the sensitivity and specificity of type 4 pattern corresponded to atrophic gastritis with HRME 90\% and 96\%, respectively [18]. In our study, when type 3-4-5 patterns were noted in the antrum, the PPV of HRME was $92.9 \%$, while the PPV of the HRME + FICE technique was $97.3 \%$. The PPV of HRME with type B1-B2-B3-A1-A2 patterns in the corpus was $84.2 \%$, while this rate was as high as $88.8 \%$ with the HRME + FICE technique. However, it is difficult to say that normal mucosal patterns observed with HRME both in the antrum and corpus are not associated with $\mathrm{H}$. pylori, because NPV in the detection of $\mathrm{H}$. pylori with HRME in the antrum was $46.8 \%$ and in the corpus $71.4 \%$. In other words, HRME can misdiagnose nearly half of the cases without $H$. pylori in the antrum and one fourth of the cases in the corpus. However, when HRME is used together with FICE, NPV was $82.7 \%$ in detecting $H$. pylori in the antrum and $96 \%$ in the corpus.

A new pattern, which we thought to be associated with intestinal metaplasia and especially observed in FICE configurations defined as 3-7 and 8 independently from the underlying mucosal pattern and evaluated by us as type 5, was observed in the antrum. While this appearance could not be observed in any patient with HRME, it was observed in nine patients with HRME + FICE, and eight of these patients were seen to have intestinal metaplasia. While a correlation was also seen between HRME + FICE appearances and severity of histopathologically detected intestinal metaplasia, such a correlation was not seen with HRME. In previous 
studies with $\mathrm{NBI}$, it was thought that visualisation of the structure named light blue crest (LBC) in the mucosa was important in detecting preneoplastic lesions in the stomach, such as intestinal metaplasia [20]. In the study conducted with $\mathrm{NBI}$, the sensitivity of LBC sign in showing the diagnosis of intestinal metaplasia was $89 \%$ and its specificity was $93 \%$. The PPV of this sign was $91 \%$ and NPV was $92 \%$ [20]. In our study, type 5 pattern was not seen in any patient in the antrum with HRME, while type 5 pattern was noted in 8 of 16 cases and type 3 and 4 patterns in seven of 16 cases with intestinal metaplasia in the antrum with HRME + FICE. In light of these data, we can say that especially type 5 pattern and type 3 and 4 patterns, which are markers of inflammation, can be guiding signs for intestinal metaplasia. With the HRME + FICE method, the sensitivity of showing intestinal metaplasia in the antrum with type 5 pattern was found to be $50 \%$, specificity was $98.8 \%$, PPV was $88.8 \%$, and NPV was $91.5 \%$. Sensitivity and specificity of A1 and A2 patterns thought to be closely associated with atrophy and intestinal metaplasia in the corpus were 33.3\% and $89.9 \%$, respectively, for HRME, while the sensitivity and specificity with HRME + FICE were $80 \%$ and $98.8 \%$, respectively. Considering the results obtained, we can say that HRME is not an efficient method for demonstrating intestinal metaplasia in both the antrum and the corpus. On the other hand, use of the HRME + FICE technique is seen to make a worthwhile contribution to the demonstration of intestinal metaplasia both in the antrum and corpus. We can say that previously undefined type 5 pattern, particularly in the antrum, carries
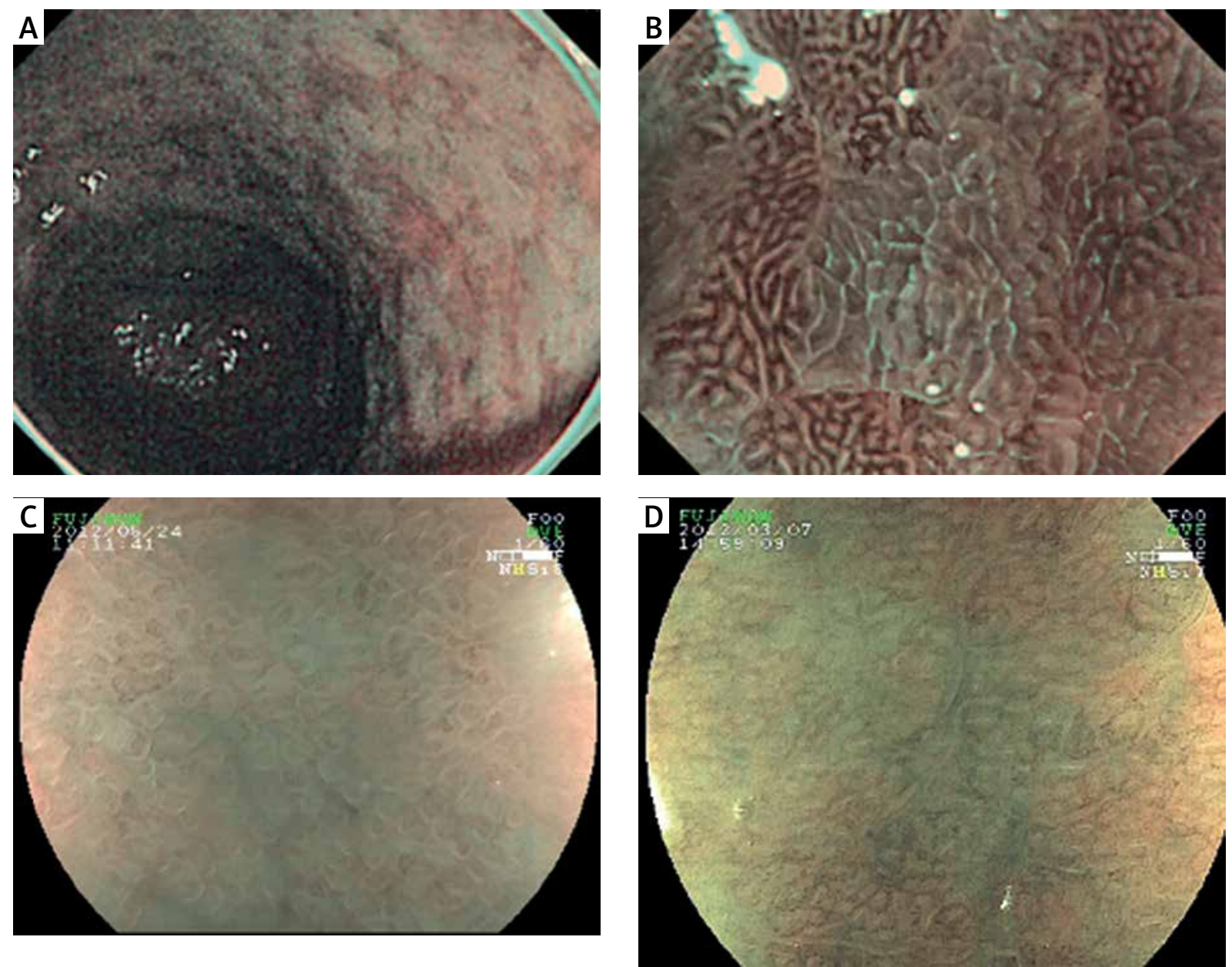

Figure 1. LBC and "patchy appearance" demonstrations with NBI and HRME + FICE. A - LBC image in the stomach with NBI. B - LBC image with magnified NBI. C, D - Gastric mucosa images with intestinal metaplasia with "patchy appearance" independent of the underlying pattern type in the antrum with HRME + FICE $\angle B C$ - light blue crest, NBI - narrow band imaging, HRME + FICE - high-resolution magnified endoscopy + flexible spectral imaging colour enhancement. 
a high PPV value like $88.8 \%$, and in the presence of this appearance and in cases with type 3-4 patterns associated with inflammation biopsy should be carried out for intestinal metaplasia. In addition, we can also say that LBC sign identified with NBI imaging is seen as patchy areas in the HRME + FICE method, and the appearance can be data with high specificity in the detection of intestinal metaplasia (Figure 1). This should be supported with clinical studies involving greater numbers of patients.

Chromoendoscopic techniques continue to develop in recent years too. Another newly developed technique is the image-enhanced endoscopy (IEE) method. This method, called LASEREO (Fujifilm Co., Japan) provides detailed imaging under laser light. In the study of $\mathrm{Na}$ kashima et al. sensitivity and specificity in detecting $H$. pylori with white light endoscopy were $66.7 \%$ and $60 \%$, respectively [21].

According to the results obtained from our study, the HRME + FICE method provides additional contribution to HRME for the detection of $H$. pylori presence and diagnosis of chronic gastritis caused by it, as well as showing intestinal metaplasia both in the antrum and the corpus. Sensitivity and specificity of the correlation of the method with the presence of $H$. pylori and chronic inflammation, especially in the corpus, are rather high, and it looks like a method that will substantially decrease the need for biopsy for H. pylori. Nevertheless, it should be taken into account that the patient group was selected from patients not using NSAIDs and/or proton pump inhibitors. Data are not available about the results that would be obtained in this group of patients following eradication therapies.

While previously four types of mucosal patterns were identified with magnified endoscopy and chromoendoscopy methods in the antrum, we identified an additional appearance pattern with the HRME + FICE technique. We noted that this pattern was especially closely associated with intestinal metaplasia independently of the underlying appearance pattern in the mucosa, and this appearance pattern was highly predictive of intestinal metaplasia.

\section{Conclusions}

In light of the results of this study comparing gastric mucosal structure with HRME and HRME + FICE techniques, it can be said that the HRME + FICE method provides an additional diagnostic contribution to HRME in showing $H$. pylori, intestinal metaplasia, and chronic inflammation and has higher sensitivity and specificity. The "patchy appearance", not previously identified with FICE in the evaluations of gastric mucosa and noted in the antrum probably due to mucosal thinning induced submucosal vascular areas, can be a guiding sign especially for intestinal metaplasia. Although this study is the first study evaluating gastric mucosal structure and the presence of $H$. pylori with the HRME and HRME + FICE techniques, new clinical studies with increased numbers of patients and comparing with chromoendoscopy including dyeing techniques are needed.

\section{Conflict of interest}

The authors declare no conflict of interest.

\section{References}

1. Wasielica-Berger J, Baniukiewicz A, Wroblewski E, et al. Magnification endoscopy and chromoendoscopy in evaluation of specialized intestinal metaplasia in Barrett's esophagus. Dig Dis Sci 2011; 56: 1987-95.

2. Hirata M, Tanaka S, Oka S, et al. Magnifying endoscopy with narrow band imaging for diagnosis of colorectal tumors. Gastrointest Endosc 2007; 65: 988-95.

3. Kiesslich R, Von Bergh M, Hahn M, et al. Chromoendoscopy with indigocarmine improves the detection of adenomatous and nonadenomatous lesions in the colon. Endoscopy 2001; 33: 1001-6.

4. Dinis-Ribeiro M, da Costa-Pereira A, Lopes C, et al. Magnification chromoendoscopy for the diagnosis of gastric intestinal metaplasia and dysplasia. Gastrointest Endosc 2003; 57: 498-504.

5. Cho WY, Jang JY, Lee DH, et al. Recent advances in image-enhanced endoscopy. Clin Endosc 2011; 44: 65-75.

6. Kiesslich R, Fritsch J, Holtmann M, et al. Methylene blue-aided chromoendoscopy for the detection of intraepithelial neoplasia and colon cancer in ulcerative colitis. Gastroenterology 2003; 124: 880-8.

7. Kiesslich R, Goetz M, Angus EM, et al. Identification of epithelial gaps in human small and large intestine by confocal endomicroscopy. Gastroenterology 2007; 133: 1769-78.

8. Marion JF, Waye JD, Present DH, et al. Chromoendoscopy-targeted biopsies are superior to standard colonoscopic surveillance for detecting dysplasia in inflammatory bowel disease patients: a prospective endoscopic trial. Am J Gastroenterol 2008; 103: 2342-9.

9. Akarsu M, Akarsu C. Evaluation of new technologies in gastrointestinal endoscopy. J Soc Laparoendosc Surg 2018; 22: pii:e2017.00053.

10. Singh R, Lee SY, Vijay N, et al. Update on narrow band imaging in disorders of the upper gastrointestinal tract. Dig Endosc 2014; 26: 144-53.

11. Tatsuta M, lishi H, Ichii M, et al. Chromoendoscopic observations on extension and development of fundal gastritis and intestinal metaplasia. Gastroenterology 1985; 88: 70-4.

12. Yagi K, Watanabe J, Nakamura A, et al. Magnifying views of gastritis - AB classification. Stomach Intestine 2007; 42: 697704.

13. Redéen S, Petersson F, Jönsson KÅ, et al. Relationship of gastroscopic features to histological findings in gastritis and Helicobacter pylori infection in a general population sample. Endoscopy 2003; 35: 946-50. 
14. Mihara M, Haruma K, Kamada T, et al. The role of endoscopic findings for the diagnosis of Helicobacter pylori infection: evaluation in a country with high prevalence of atrophic gastritis. Helicobacter 1999; 4: 40-8.

15. Gonen C, Simsek I, Sarioglu S, et al. Comparison of high resolution magnifying endoscopy and standard videoendoscopy for the diagnosis of Helicobacter pylori gastritis in routine clinical practice: a prospective study. Helicobacter 2009; 14: 12-21.

16. Serin A, Tankurt E, Şarkış C, et al. The prevalence of Helicobacter pylori infection in patients with gastric and duodenal ulcers - a 10-year, single-centre experience. Gastroenterology Rev 2015; 10: 160-3.

17. Shin WG, Kim HU, Song HJ, et al. Surveillance strategy of atrophic gastritis and intestinal metaplasia in a country with a high prevalence of gastric cancer. Dig Dis Sci 2012; 57: 746-52.

18. Anagnostopoulos G, Yao K, Kaye P, et al. High-resolution magnification endoscopy can reliably identify normal gastric mucosa, Helicobacter pylori-associated gastritis, and gastric atrophy. Endoscopy 2007; 39: 202-7.

19. Yagi K, Nakamura A, Sekine A. Magnifying endoscopy of the gastric body: a comparison of the findings before and after eradication of Helicobacter pylori. Dig Endosc 2002; 14: 76-82.

20. Uedo $\mathrm{N}$, Ishihara R, lishi $\mathrm{H}$, et al. A new method of diagnosing gastric intestinal metaplasia: narrow-band imaging with magnifying endoscopy. Endoscopy 2006; 38: 819-24.

21. Nakashima $\mathrm{H}$, Kawahira $\mathrm{H}$, Kawachi $\mathrm{H}$, et al. Artificial intelligence diagnosis of Helicobacter pylori infection using blue laser imaging-bright and linked color imaging: a single-center prospective study. Ann Gastroenterol 2018; 31: 462-8.

Received: 21.12 .2018

Accepted: 14.02.2019 